\title{
Neural network prediction model of the pilots' errors
}

\author{
A.N. Danilenko ${ }^{1}$

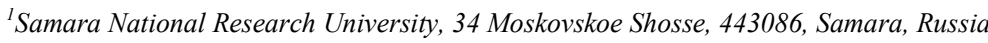

\begin{abstract}
This paper introduces a hybrid model of the neuro-fuzzy classifier with an integrated prediction of pilots' mistakes. Experiments and studies of the network were conducted on real and test samples. The upgraded hybrid neuro-fuzzy classifier structure and the learning algorithm can solve the problem of the need for multiple individual performance measurements, the dynamics of which would make it possible to build a trend and solve the problem on small samples. Used in organizational and management activities, this principle can help in predicting the danger caused by the human factor.
\end{abstract}

Keywords: forecast; wrong actions of the pilot; intellectual support; hybrid neuro-fuzzy classifier; two-layer perceptron; small samples

\section{Introduction}

Throughout the history of aviation, pilot's error as a safety-reducing factor was the subject of attention of the flight-technical and airlines management personnel and of the researchers in the field of psychology. For a long time, claiming the pilot's error, made him guilty of the task failure, of the equipment damage and, eventually, of his death. Therefore, all measures to combat errors were aimed at professional selection, training and «education» (punishment). These measures are obviously necessary, but not sufficient, since the errors are committed by highly-qualified pilots, which suggests that the human factor accidents are not limited to the issue of professional incompetence.

Pilot's activities feature unusual for other professions spatial orientation. The pilot evaluates the aircraft position in space according to the visual reference from the ground and from electronic devices, and in bad weather and visibility conditions only from instruments. The quality of pilot-instruments interaction is largely determined by his individual psychological characteristics.

Psychological characteristics of the person are one of the main causes of air accidents. In this case, the role of self-esteem, stress levels and their influence on the pilots' professional qualities are the most interesting aspects in the error occurrence [1].

\section{The object of the study}

The study was conducted in the air squadron in one part of the closed garrison. Based on the data of psychological testing, we have created a pilot's professional efficiency diagnostic forecast complex.

\subsection{Mathematical formulation of the problem}

Every person can be described by a finite set of attributes [2], in this case, the characteristics obtained by the psychodiagnostics, $\mathrm{A}=\left\{\mathrm{A}_{1}, \mathrm{~A}_{2}, \ldots, \mathrm{A}_{\mathrm{n}}\right\}$, where $n=\overline{1,55}$. Each of $\mathrm{A}_{\mathrm{i}}$ corresponds to a universal set of $\mathrm{U}_{\mathrm{i}}$, consisting of linguistic variables and numerical values $\left\{\mathrm{a}_{1 \mathrm{i}}, \mathrm{a}_{2 \mathrm{i}}, \ldots \mathrm{a}_{\mathrm{nii}}\right\}$, where $i=\overline{1, n}$.

In turn, each element of the function has its own identity.

The result set $\mathrm{R}=\left\{\mathrm{R}_{1}, \mathrm{R}_{2}, \ldots, \mathrm{R}_{\mathrm{k}}\right\}$, where $k=\overline{1,4}$.

$\mathrm{R}=\{$ not suitable, partly suitable, mainly suitable, suitable $\}$

The fuzzy base of rules in general will look as follows:

$$
\begin{aligned}
& \text { IF }\left(A_{1}=a_{i 11}^{1}, A_{2}=a_{i 22}^{1}, \ldots, A_{n}=a_{i n n}^{1}\right) \text { then }\left(\mu_{R 1}=\mu_{1}^{1}, \mu_{R 2}=\mu_{2}^{1}, \ldots, \mu_{R k}=\mu_{k}^{1}\right) \\
& \text { IF }\left(A_{1}=a_{i 11}^{2}, A_{2}=a_{i 22}^{2}, \ldots, A_{n}=a_{i n n}^{2}\right) \text { then }\left(\mu_{R 1}=\mu_{1}^{2}, \mu_{R 2}=\mu_{2}^{2}, \ldots, \mu_{R k}=\mu_{k}^{2}\right) \\
& \ldots \\
& \text { IF }\left(A_{1}=a_{i 11}^{r}, A_{2}=a_{i 22}^{r}, \ldots, A_{n}=a_{i n n}^{r}\right) \text { then }\left(\mu_{R 1}=\mu_{1}^{r}, \mu_{R 2}=\mu_{2}^{r}, \ldots, \mu_{R k}=\mu_{k}^{r}\right)
\end{aligned}
$$

where $\mu_{R l}$ - the degree of the rule belonging to $\mathrm{R}_{1}$ class.

\section{Methods}

Regression analysis appears to be the traditional method of forecasting in psychology. It is assumed that the values of the time series is a random time function, and the task is to identify the correct model. The choice of the form of the function is not formalized and depends entirely on the expert's experience. At the same time, a neural network acts as a universal approximator of the training data, so the use of neural networks for the prediction is very promising.

In addition, the neural network can be seen as an adaptive model, as it can develop while gaining new information. Human behavior by nature is evolutional, and the use of static models leads to the forecast quality deterioration.

Another problem that we faced was the need for a large amount of input data for network training. It is usually assumed that the time series contains at least hundreds of values, and it is impossible for us to complete this amount of observations. However, 
there are opportunities to train the neural network on small amounts of input data. In this case, the a repeated learning on the same examples is being used, as well as different methods of time series processing, allowing to extend the training set.

The peculiarity of the problem lies in the fact that self-assessment and stress levels cannot be the input vector for the prediction of the network. The input is the values vector of the professional suitability dynamics for the period from six months to two years (that means, from 3 to 12 measurements, testing being conducted no more frequently than once every two months). By the professional suitability dynamics of the candidate we mean the degree of affiliation to one of four classes: the candidate fully meets the requirements of the specialty, basically corresponds, partially meets or does not meet - which in turn is obtained by analysis of 55 psychological characteristics.

Since the information, based on which a decision on the professional suitability of the candidate is made, is the result of various psychological techniques, classified data should be inaccurate or poorly defined. Due to this fact, it is necessary to use fuzzy logic and fuzzy sets theory as an effective approach to solving this problem.

To solve all the problems above, a modified hybrid model of neuro-fuzzy classifier with an integrated forecast function has been developed.

\subsection{Hybrid neuro-fuzzy classifier}

San and Jang offered the architecture to solve the fuzzy classification problem[3]. One possible structure of a hybrid neurofuzzy classifier is shown in Fig. 1.

Neuro-fuzzy network consists of four layers.

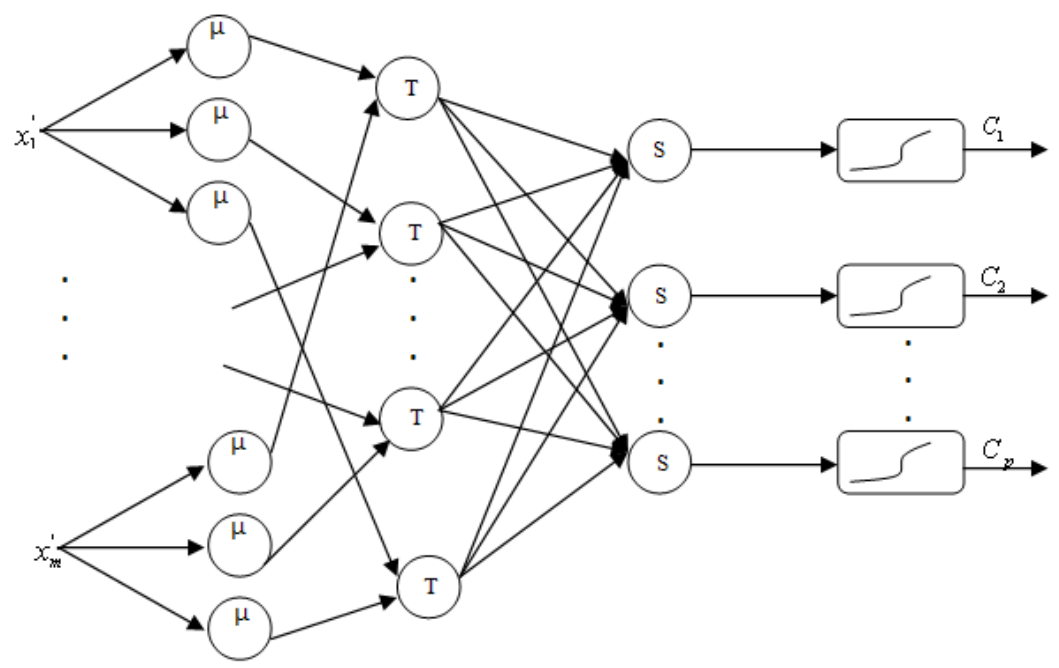

Fig. 1. Structure of the neuro-fuzzy classifier.

First-layer-elements implement the fuzzification operation, in other words, they form the degree of the membership of input data for the defined fuzzy sets $A_{i j}$

$$
\mu_{A_{j i}}\left(x_{j}^{\prime}\right)=\exp \left[-\frac{1}{2}\left(\frac{x_{j}^{\prime}-c_{i j}}{\sigma_{i j}}\right)^{2}\right]
$$

where $c_{i j}, \sigma_{i j}$ - the parameters of the membership bell-shaped type function.

The initial values of these parameters are set so as membership function satisfies the completeness, normality and convexity properties. Values should be equally distributed in the input vectors $X$. The values of these parameters can be adjusted in the process of the network education, which is based on the gradient method.

Each element of the second layer is an "I" neuron. It performs the aggregation of each database rule prerequisites truth degrees according to the T-norm operation using the following formulas:

$$
\begin{aligned}
& \alpha_{1}=\min \left\{A_{11}\left(x_{1}\right), A_{12}\left(x_{2}\right), \ldots, A_{1 n}\left(x_{n}\right)\right\} \\
& \alpha_{2}=\min \left\{A_{21}\left(x_{1}\right), A_{22}\left(x_{2}\right), \ldots, A_{2 n}\left(x_{n}\right)\right\} \\
& \ldots \\
& \alpha_{n}=\min \left\{A_{n 1}\left(x_{1}\right), A_{n 2}\left(x_{2}\right), \ldots, A_{n n}\left(x_{n}\right)\right\}
\end{aligned}
$$

Third-layer elements perform the aggregation of each database rule prerequisites truth degrees according to the S-norm operation.

To solve the problem of candidates' classification for vacancies, basing on psycho-diagnostics, an input volume is quite small, with an average of 50 values. In order to speed up the network training algorithm and its simplifications, we should replace neurons of the third layer with the neurons that perform normalization and calculate the following values:

$$
\beta_{1}=\frac{\alpha_{1}}{\alpha_{1}+\alpha_{2}+\ldots+\alpha_{n}}
$$




$$
\begin{aligned}
& \beta_{2}=\frac{\alpha_{2}}{\alpha_{1}+\alpha_{2}+\ldots+\alpha_{n}} \\
& \ldots \\
& \beta_{n}=\frac{\alpha_{n}}{\alpha_{1}+\alpha_{2}+\ldots+\alpha_{n}}
\end{aligned}
$$

The elements of the fourth layer are used to calculate the conclusion values for each rule:

$$
\begin{aligned}
& y_{1}^{\prime}=B_{1}^{-1}\left(\alpha_{1}\right)=a_{1}+\frac{1}{b_{1}} \ln \frac{1-\alpha_{1}}{\alpha_{1}} \\
& y_{2}^{\prime}=B_{2}^{-1}\left(\alpha_{2}\right)=a_{2}+\frac{1}{b_{2}} \ln \frac{1-\alpha_{2}}{\alpha_{2}} \\
& \cdots \\
& y_{n}^{\prime}=B_{n}^{-1}\left(\alpha_{n}\right)=a_{n}+\frac{1}{b_{n}} \ln \frac{1-\alpha_{n}}{\alpha_{n}}
\end{aligned}
$$

where $a_{i}, b_{i}$ - nonlinear membership function parameters $\mu_{B_{i}}(y)$ to the rule conclusion fuzzy sets.

Fuzzy network outputs are computed as follows: $y_{i}^{\prime}=\beta_{i} B_{i}^{-1}\left(\alpha_{i}\right)$.

These outputs are interpreted as the membership degree of the object to the corresponding class. Since hybrid neuro-fuzzy classifier is represented as a multi-layer structure with a direct signal spread and the output variable value can be changed by adjusting the parameters of elements in layers, the gradient algorithms can be used to train the network.

Using this neuro-fuzzy network model the problem of classification can be solved, the results of which are the input vector for the prediction network.

\subsection{The network structure}

Then, a conventional two-layer perceptron can be added to a modified hybrid neuro-fuzzy classifier [4] with an additional neuron, which accumulates input values for classification prediction vector and, in fact, is one of Grossberg star [5] (Fig. 2). Two-layer perceptron was implemented without any changes.

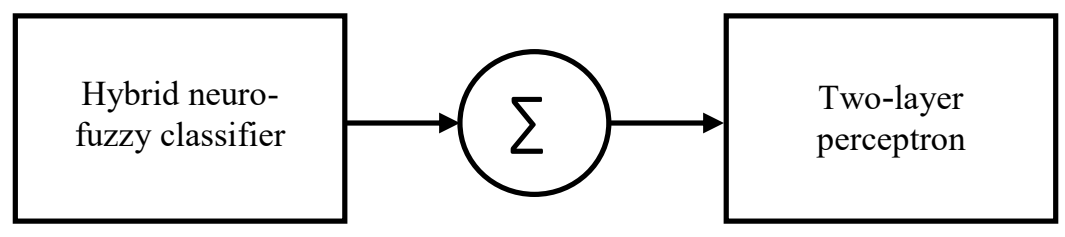

Fig. 2. The network structure.

\section{Results and discussion}

Tsukomoto algorithm was implemented in the hybrid neuro-fuzzy classifier, as well as a backpropagation method - a learning algorithm. The influence of the hybrid neuro-fuzzy classifier was also detected. The optimal structure of the network was selected: the volume of training sample - 35 samples, one network learning step $\mathrm{h}=0,45$ and Gaussian fuzzification

\begin{tabular}{|c|c|c|c|c|c|}
\hline \multirow{2}{*}{ The number of inputs } & \multicolumn{4}{|c|}{$\mathrm{Kn} / \mathbf{s}$} & \multirow{2}{*}{ Total } \\
\hline & $1 \%$ & $5 \%$ & $10 \%$ & $15 \%$ & \\
\hline 2 & 12 & 7 & 1 & 2 & 22 \\
\hline 3 & 3 & 8 & 9 & 11 & 31 \\
\hline 4 & 0 & 0 & 3 & 1 & 4 \\
\hline 5 & 0 & 0 & 2 & 1 & 3 \\
\hline $\begin{array}{l}\text { The number of neurons in the } \\
\text { hidden layer }\end{array}$ & $1 \%$ & $5 \%$ & $10 \%$ & $15 \%$ & Total \\
\hline 2 & 13 & 15 & 11 & 10 & 49 \\
\hline 3 & 2 & 0 & 3 & 4 & 9 \\
\hline 4 & 0 & 0 & 1 & 1 & 2 \\
\hline
\end{tabular}
function, defuzzification method based on the r.m.s. deviations.

A study of the prediction quality using the constructed neural network was conducted on the test and the real-time series. For each time series, a structure of the network was chosen, providing the best quality of forecasting. The results are shown in Table 1.

Table 1. The frequency of the various structures use for the two-layer perceptron.

The prediction is considered to be sufficiently accurate, if the prediction error is not more than $20 \%$. 
Fig. 3 shows the dependence of the prediction accuracy on the noise effects using different methods.

The "ideal" forecast - a forecast, the values deviation of which is caused only by random factors. If the prediction error is slightly different from the error of the "ideal" forecast, then it can be considered accurate.

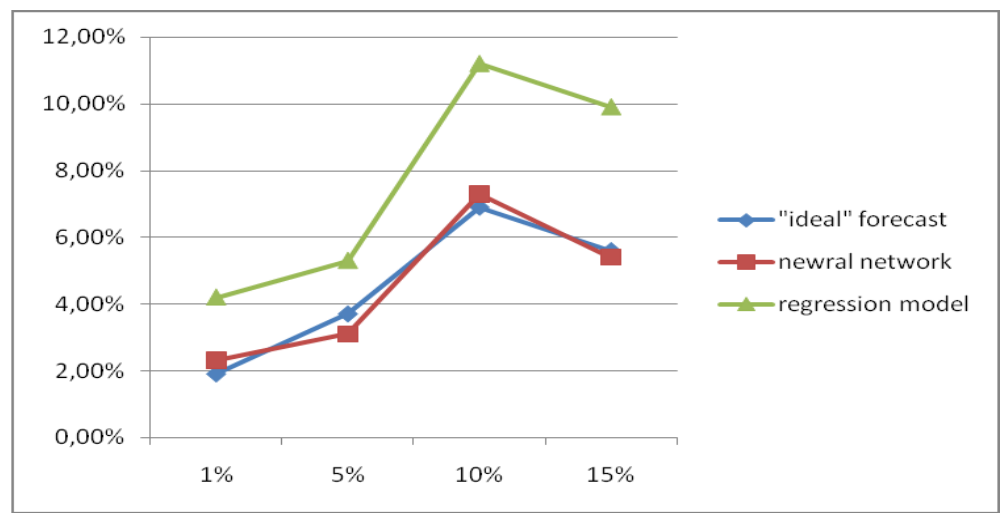

Fig. 3. Dependence of the prediction accuracy on the noise effects.

$$
\begin{aligned}
& K 1=\frac{\sigma_{\varepsilon}^{2}}{\sigma_{D}^{2} \cdot 100 \%}-\sqrt{K 2=\sqrt{\frac{\sum_{t=n+1}^{n+l}\left(Y_{t}^{*}-Y_{t}\right)^{2}}{\sum_{t=n+l}^{n+l} Y_{t}^{2}+\sum_{t=n+l}^{n+l}\left(Y_{t}^{*}\right)^{2}}} \text { - inconsistency coefficient (the second Teil coefficient), estimating the forecast accuracy. }}
\end{aligned}
$$

\section{Conclusion}

According to the study it can be concluded that the predictions, obtained using a neural network, have high level of accuracy and for many dynamics types seem to be significantly superior to the ones obtained using the regression model.

Moreover, the upgraded hybrid neuro-fuzzy classifier structure and the learning algorithm can solve the problem of the need for multiple individual performance measurements, the dynamics of which would make it possible to build a trend and solve the problem on small samples.

This approach allows with a certain degree of probability to calculate a predisposition to wrong actions in each case. If used in organizational and management activities, this principle can help in predicting the danger caused by the human factor.

\section{References}

[1] Danilenko AN, Ihsanova SG, Komakov VV. The diagnostic prediction of the professional performance of the pilot. Moscow: Mechanical engineering Flight 2012; 7: 53-60

[2] Novak V, Perfilieva I, Mochkorzh I. Mathematical Principles of Fuzzy Logic. Moscow: Fizmatlit, 2006; 252 p.

[3] Borisov VV, Kruglov VV, Fedulov AS. Indistinct models and networks. Moscow: The hot line-Telecom, 2007; 284 p.

[4] Osovsky S. Neural networks for information processing. Moscow: Finance and Statistics, 2002; 344 p. 